\title{
Correction to: Precautionary replenishment in financially-constrained inventory systems subject to credit rollover risk and supply disruption
}

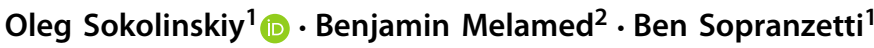

Published online: 18 August 2018

(c) Springer Science+Business Media, LLC, part of Springer Nature 2018

\section{Correction to: Annals of Operations Research https://doi.org/10.1007/s10479-018-2965-1}

The original version of this article was revised as some author corrections were overlooked by vendor.

The original article can be found online at https://doi.org/10.1007/s10479-018-2965-1.

$凶$ Oleg Sokolinskiy

oleg.sokolinskiy@business.rutgers.edu

Benjamin Melamed

melamed@business.rutgers.edu

Ben Sopranzetti

sopranze@business.rutgers.edu

1 Department of Finance and Economics, Rutgers Business School - Newark and New Brunswick, Rutgers University, 100 Rockafeller Rd., Piscataway, NJ 08854, USA

2 Department of Supply Chain Management, Rutgers Business School - Newark and New Brunswick, Rutgers University, 100 Rockafeller Rd., Piscataway, NJ 08854, USA 